\title{
MONTE CARLO STUDY OF A PERIODICALLY PINNED 2D SUPERCONDUCTOR
}

\section{N. Rezlescu, R. Anghelache and I. Crobotaru}

Institute of Technical Physics, Mangeron ave. 47, 6600 Iasi, Romania

We show that a natural assumption on the moving mechanism of vortices in a periodically pinned, two-dimensional superconductor in the mixed state is the basis for a $n$-clock-like model. The pinning points are considered to be centered in each unit cell of the superconducting lattice. This property enables one to use a discrete version of the $X Y$ model used in the simulations focusing on the melting of the Abrikosov lattice of vortices in a system of Josephson arrays. A Monte Carlo study performed on the corresponding Hamiltonian in a field given by a filling factor of $f=1 / 2$ exhibits two phase transitions, at the temperatures $T_{\mathrm{c} 1} \approx 0.325$ and $T_{\mathrm{m}} \approx 0.65$. The numerical estimation of the Challa parameters for both transitions indicates their continuous nature. The physical meaning of the lower transition is the expulsion of the magnetic field from the superconducting system, i.e. the Meissner effect. The physical equivalent of the upper transition is the melting of the pinned vortex lattice.

PACS numbers: 64.60.Fr, 74.25.Bt, 75.10.Hk

One method of studying the transition from the normal phase to the Abrikosov phase is the Monte Carlo approach on a classical 2D $X Y$ model applied to a Josephson coupled array of superconducting grains [1-6]. Our work is based on the belief that the presence of the pinning requires a discreteness of the numerical model used in the simulations [7-10].

We have shown elsewhere $[11,12]$ that the influence of the periodicity of the pinning centers on a flux line lattice can be simulated by means of a planar Potts (clock) model [13]. Briefly, the effective pinning will make the vortex movements discontinuous, as opposed to a pin-free superconductor, where the vortices can move continuously. The appropriate $X Y$ model for the frustrated flux line lattice is [3]

$$
H=-\sum_{i, j} J_{i, j} \cos \left(\phi_{i}-\phi_{j}-A_{i, j}\right),
$$

where $J_{i j}=J$ (constant) is the Josephson coupling between the superconducting grains, labelled $i$ and $j, \phi_{i, j}$ are the corresponding phases of the superconducting order parameter at sites $i$, respectively $j$, and

$$
A_{i j} \equiv(2 e / \hbar c) \int_{i}^{j} \boldsymbol{A}(l) \cdot \mathrm{d} l,
$$

with $\boldsymbol{A}$ being the vector potential $(\boldsymbol{B} \equiv \boldsymbol{\nabla} \times \boldsymbol{A})$. 
Considering a lattice of superconducting grains, the sum of the order parameter's phases around a planar plaquette equals $2 \pi(m-f)$. Here $m=1,0,-1$ corresponds to the presence of a vortex, absence of it and, respectively, presence of an antivortex in the plaquette, $f \equiv B / \Phi$ is the filling factor in an applied field, $\Phi \equiv 2 e / \hbar c$ is the flux quanta and $B-$ the local magnetic field.

The presence of a pinned, isolated vortex, in the center of a unit cell in a quadratic superconducting lattice, will give equal (for reasons of symmetry) phase differences (PD) on the bonds, i.e. $2 \pi(1-f) / 4$. In this paper we have chosen $f=1 / 2$, thus, the corresponding bond phase differences will be $\pm \pi / 4$, if there is an isolated vortex and, respectively, zero if such an object is not present. We make the natural assumption that if a second pinned vortex becomes the neighbor of the first, the PD on the bond common to the both occupied cells will become zero, and the total PDs around each of these neighbor cells will repartition equally on each group of the three uncommon bonds, so the PDs will become $\pi / 3$. Following this rule, the presence of a third neighboring vortex will give for the PDs a value of $\pi / 2$.

This mechanism can be described by a restricted $n$-clock model,

$$
H=-J \sum_{i, j} \cos \left(\phi_{i, j} / n\right),
$$

with $n$ being determined by the symmetry of the superconducting lattice and the filling factor (i.e. the applied magnetic field). The particular set of possible values for the PDs $\left(\phi_{i, j} / n\right)$, i.e. $\pm \pi / 4, \pm \pi / 3, \pm \pi / 2$, and 0 , are given by the particular choice of the applied field, i.e. $f=1 / 2$. In general, the maximum number of possible values for the PDs is determined by the chosen dynamics of the vortices (the mechanism chosen here makes available only four different values to add in the Hamiltonian from each bond), and the actual values of the PDs are given by the filling factor. Thus, the model Hamiltonian (1) becomes a modified (restricted) $n$-clock model in "zero field", based on the restricted set of values for the PDs (here $n$ results to be 12 ).

We have used a 2D version of the model (1), that is, the centers of pinning are perfectly ordered in a lattice dual to that of the superconducting grains. This model should fit, in principle, to 2D superconducting layer.

We performed Monte Carlo simulations using the Metropolis algorithm on planar lattices of size $L^{2}, L=40 \div 80$, in the canonical ensemble based on Hamiltonian (1), using periodic boundary conditions. We needed $3 \div 5 \times 10^{4}$ Monte Carlo steps per lattice (MCSL) to achieve the thermal equilibrium (that is, $3 \div 5 \times 10^{4} \mathrm{~L}^{2}$ Monte Carlo steps per "spin") and $12 \div 15 \times 10^{4}$ MCSL for averaging. We have calculated the internal energy and the expectation value of the order parameter (chosen here to be the thermal average of $\sin \left(\phi_{i, j} / n\right)$ ) and the corresponding $2 n d$ and 4 th order moments. The latter two are necessary to calculate the Binder (order parameter, $P$ ) and Challa (internal energy, $E)$ cumulants $U_{P, E} \equiv 1-\left\langle X^{4}\right\rangle /\left(3\left\langle X^{2}\right\rangle^{2}\right)$, where $X \equiv P, E$ respectively [14].

This system exhibits three thermodynamical phases. The PDs are zero valued in the low temperature phase (no vortices are present in the sample). Above the lower critical temperature $T_{\mathrm{c} 1}$, the vortices enter in the sample through a second-order transition and an ordered middle phase is developed. In this state 


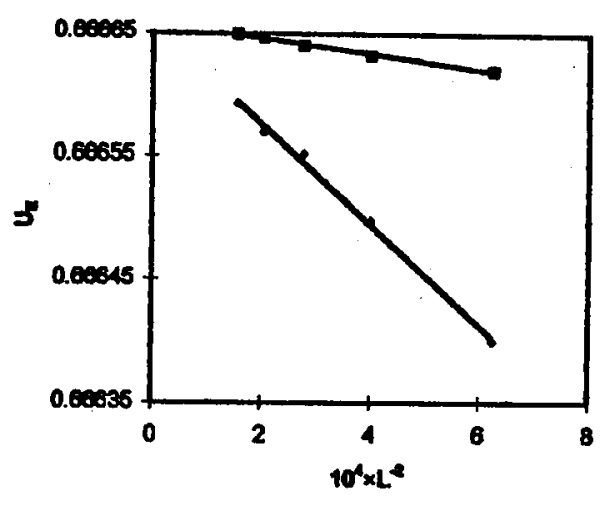

Fig. 1. Values of the Challa parameter, $U_{E}$, versus $L^{-2}$, for both transitions; $L=80$ (squares $-T_{\mathrm{c} 1}$, rhombs $-T_{\mathrm{m}}$ ).

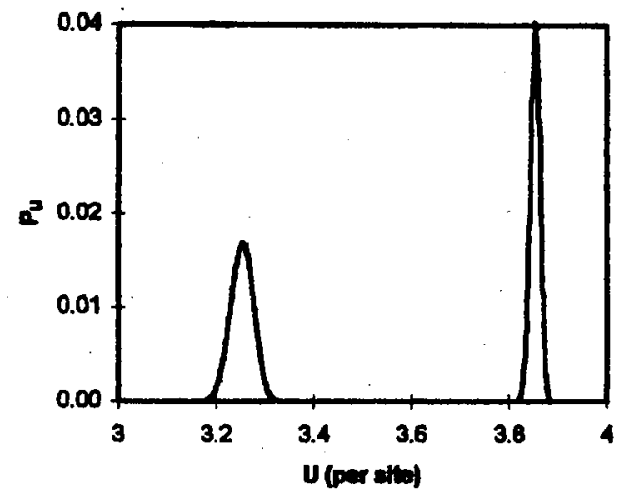

Fig. 2. Probability distributions for the internal energy at $T_{\mathrm{c} 1}$ (at higher energies) and $T_{\mathrm{m}}$ (at lower energies); $L=80$.

the PDs have $\pm \pi / 4$ values (they form a lattice of vortices as in the mixed superconducting state). Above a temperature $T_{\mathrm{m}}$ this mixed state undergoes a continuous phase transition to the high temperature disordered one. The continuous character of both transitions is determined by the values of the Challa parameter found to be, in the limit of infinite $L, 0.66665(9)$ for the low temperature transition and, respectively, $0.66665(6)$ for the higher temperature one, the data for both transitions are displayed in Fig. 1. The continuous nature of the transitions is also confirmed by the single peaked probability distribution of the internal energy $\left(6 \times 10^{6} \mathrm{MCSL}\right.$, $L=80$ ) (Fig. 2). Using the cumulant-crossing method on the Binder parameter [15], we were able to determine $T_{\mathrm{m}}=0.65(2)$. The temperature is written here in $J / k_{\mathrm{B}}$ units, where $k_{\mathrm{B}}$ is the Boltzmann constant. We could not use the same method of determination of the critical temperature $T_{\mathrm{c} 1}$, however, the values found for $T_{\mathrm{c} 1}$ (taken here as the temperatures corresponding to the peaks in specific heat) in the simulations are less than 0.325 and very slow (i.e. $\alpha \approx 0$, where $\alpha$ is the critical coefficient for the specific heat) increasing with $L$. 
For lower applied fields we expect that a model similar to (1), with very few (isolated) vortices, could be relevant for the study of the flux line lattice (FLL) melting. The limiting case is that the PDs will fluctuate in steps of $\pm \pi / 2$, thus, our model becomes a 2D Ising model in zero applied field, consequently exhibiting a single second-order phase transition.

We found that a restricted planar Potts ( $n$-clock) model can describe the local magnetic field fluctuations in the high- $T_{c}$ superconductors with periodic pinning. The value of $n$ is determined by the superconducting grains lattice symmetry and the external applied field. The number of possible values of the terms entering into the Hamiltonian are determined by the moving mechanism of the neighboring vortices. Two continuous transitions are found. The phase between the transitions is equivalent to the mixed phase in the type-II superconductors. The low temperature ordered phase is physically equivalent to the Meissner state. The fluctuations of the local magnetic field due to the vortex movement is found to play an essential role in the flux line lattice melting transition. The continuous nature of the melting transition is given by the ordering of the centers of pinning as suggested by theory [4] and experiment [16].

\section{References}

[1] C. Dasgupta, B.I. Halperin, Phys. Rev. Lett. 47, 1556 (1981).

[2] Y.H. Li, S. Teitel, Phys. Rev. Lett. 65, 2595 (1990).

[3] Y.H. Li, S. Teitel, Phys. Rev. Lett. 66, 3301 (1991).

[4] R.E. Hetzel, A. Sudbo, D.A. Huse, Phys. Rev. Lett. 69, 518 (1992).

[5] M.P.A. Fisher, G. Grinstein, Phys. Rev. Lett. 60, 108 (1988).

[6] M.P.A. Fisher, D.H. Lee, Phys. Rev. B 39, 2756 (1989).

[7] D.R. Nelson, H.S. Seung, Phys. Rev. B 39, 9153 (1989).

[8] D.R. Nelson, V.M. Vinokur, Phys. Rev. Lett. 68, 2398 (1992).

[9] W.K. Kwok, J. Fendrich, U. Welp, S. Fleshler, J. Downey, G.W. Crabtree, Phys. Rev. Lett. 72, 1092 (1994).

[10] W. Jiang, N.-C. Yeh, D.S. Reed, U. Kriplani, D.A. Beam, M. Konczykowski, T.A. Tombrello, F. Hlotzberg, Phys. Rev. Lett. 72, 550 (1994).

[11] N. Rezlescu, R. Anghelache, P.D. Popa, I. Ciobotaru, Phys. Lett. A 205, 97 (1995).

[12] N. Rezlescu, R. Anghelache, P.D. Popa, I. Ciobotaru, J. Magn. Magn. Mater., in press.

[13] F.Y. Wu, Rev. Mod. Phys. 54, 235 (1982).

[14] K. Binder, in: The Monte Carlo Method in Condensed Matter Physics, Ed. K. Binder, Springer-Verlag, Berlin 1995, p. 5.

[15] M.L. Plumer, A. Mailhot, in: Computer Simulation Studies in Condensed Matter Physics VIII, Eds. D.P. Landau, K.K. Mon, H.B. Schuttler, Springer-Verlag, Berlin 1995, p. 44.

[16] W.K. Kwok, J. Fendrich, S. Fleshler, U. Welp, J. Downey, G.W. Crabtree, Phys. Rev. Lett. 72, 1088 (1994). 